\title{
Imperatorin exhibits anticancer activities in human colon cancer cells via the caspase cascade
}

\author{
YI MEI ZHENG ${ }^{1}$, AMY XIAOXU LU ${ }^{2}$, JAMES ZHENG SHEN ${ }^{2}$, AMY HO YAN KWOK $^{2}$ and WING SHING HO ${ }^{2}$ \\ ${ }^{1}$ College of Biological Science and Technology, Minnan Normal University, Zhangzhou, Fujian 363000, \\ ${ }^{2}$ School of Life Sciences, The Chinese University of Hong Kong, Shatin, Hong Kong, SAR, P.R. China
}

Received September 2, 2015; Accepted October 21, 2015

DOI: 10.3892/or.2016.4586

\begin{abstract}
Despite advances in medical treatments for colon cancer, it remains one of the leading causes of cancer-related mortality among men. Thus, more efficacious treatment strategies for colon cancer are needed. Imperatorin is one of the major ingredients present in the root of Angelica dahurica, and has been used in herbal formulations for the treatment of hypertension and cardiovascular diseases. However, the medical properties of imperatorin remain unclear. In the present study, the anti-proliferative activities of imperatorin were investigated in the HT-29 colon cancer cell line. The results showed that imperatorin significantly inhibited HT-29 colon cancer cell growth with an $\mathrm{IC}_{50}$ value of $78 \mu \mathrm{M}$. Imperatorin induced the apoptosis of colon cancer cells through upregulation of p53 and the caspase cascade. Our findings revealed that imperatorin induced cell cycle arrest in the G1 phase. The apoptotic index showed a steady increment when the imperatorin concentration was increased. The results suggest that imperatorin exerts considerable anti-proliferative activities in HT-29 colon cancer cells and highlight the potential of imperatorin as an anticancer agent for colon cancer.
\end{abstract}

\section{Introduction}

Colon cancer is one of the leading causes of cancer-related mortality. Colorectal cancer requires time to develop. Prognosis and removal of early-stage polyps can prevent the development of cancer. Early colorectal cancer exhibits no symptoms. Moreover, a cure is not possible for most patients with metastatic colorectal cancer. Chemotherapy is the most common treatment option, which can alleviate symptoms and prolong patient life. Yet, alternative medicines are required.

Correspondence to: Professor Wing Shing Ho, School of Life Sciences, The Chinese University of Hong Kong, Shatin, MMW 604, Hong Kong, SAR, P.R. China

E-mail:ws203ho@cuhk.edu.hk

Key words: imperatorin, colon cancer cells, Angelica dahurica, apoptosis
Oxidative stress can be caused by the accumulation of intracellular reactive oxygen species (ROS) through metabolism of toxic substances. Cancer cells demand high ROS concentrations to maintain their high proliferation rate due to a higher metabolic rate. However, ROS levels can be used as a checkpoint with which to monitor cell damage. The implications for ROS regulation are highly significant for cancer therapy as commonly used radiotherapy and chemotherapeutic drugs influence tumor proliferation through ROS modulation. Anticancer phytochemicals are being used to modulate the ROS level in cancer cells which is the principal mechanism by which most anticancer agents function to elicit inhibition of tumor proliferation. Imperatorin is one of the major active coumarins found in the root of Imperata cylindrica Beauv. with less than $4 \%$ in the herbal extract (1). It was reported that imperatorin exhibits many pharmacological activities including anti-inflammatory, anti-coagulant and anti-proliferative (1-3). Previous studies showed that imperatorin inhibited SNU 449 liver cancer cell growth (1). Other studies have shown that imperatorin mediates oncogene expression and induces cell cycle arrest (4-8). A recent study showed that imperatorin suppressed voltage-gate sodium channels and neuronal excitability through inhibition of the voltage-gate sodium channels (9). In the present study, we investigated the anti-proliferative activities of imperatorin in HT-29 cancer cells and its effects on signaling pathways involved in apoptosis as well as its antioxidant effects. We investigated the expression of marker proteins involved in these processes and the potential of imperatorin as an anticancer agent.

\section{Materials and methods}

Cells, chemicals and reagents. The HT-29 colon cancer cell line was obtained from the American Type Culture Collection (ATCC, Manassas, VA, USA). Acetonitrile (ACN) (E. Merck, Darmstadt Germany), methanol (RCI, Labscan, Thailand) and trifluoroacetic acid (Sigma-Aldrich, St. Louis, MO, USA) were of HPLC grade. Distilled and deionized water $\left(\mathrm{ddH}_{2} \mathrm{O}\right)$ was prepared using the Millipore water purification system (Millipore, Milford, MA, USA). All other reagents used were of analytical reagent grade purchased from Sigma-Aldrich unless stated otherwise. All antibodies were purchased from Cell Signaling Technology, Inc. (Danvers, MA, USA) except anti-caspase-8, -Bax, -p21 and -MDM2 which were obtained 
from Santa Cruz (Dallas, TX, USA). Imperatorin was purchased from Sigma-Aldrich.

MTT assay. The growth inhibitory effect of imperatorin was evaluated in colon cancer HT-29 cells. All cells were seeded onto 96 -well microtiter plates, at a density of $6 \times 10^{3}$ cells per well and allowed to incubate overnight. The cells were treated with either $0.5 \%$ DMSO [as solvent control (Ctrl)] or various concentrations of imperatorin (as treatment group) and incubated for 24,48 and $72 \mathrm{~h}$ at $37^{\circ} \mathrm{C}$, respectively. After incubation, MTT solution $(50 \mu \mathrm{l})$ was added to each well, and the plates were incubated at $37^{\circ} \mathrm{C}$ for $3 \mathrm{~h}$. Then the solution in each well was replaced by DMSO $(150 \mu \mathrm{l})$. The absorbance was measured at $540 \mathrm{~nm}$ by TECAN Infinite M200 (Tecan Group Ltd., Männedorf, Switzerland). Each treatment or control was performed in replicates of 4-6 times. Mean absorbance readings from solvent control groups were defined as having $100 \%$ viability, and the results from the treatment groups were shown as the percentage of viability relative to the controls.

Lactate dehydrogenase $(\mathrm{LDH})$ activity assay. Cells that undergo necrosis are lysed to release cytosolic LDH into culture medium. The CytoTox 96 Non-Radioactive Cytotoxicity Assay kit (Promega, Madison, WI, USA) was used to evaluate the cytotoxic effect of imperatorin on HT-29 cells by assessing the activity of LDH present in the culture medium. After a 48-h treatment of the HT-29 cells with imperatorin, the culture medium was transferred to a 96-well plate, followed by addition of an equal volume of CytoTox 96 reagent in each well. The plate was maintained in the dark at room temperature for $30 \mathrm{~min}$. The enzymatic reaction was stopped by adding an equal volume of stop solution in each well. The absorbance was measured at $490 \mathrm{~nm}$ by TECAN infinite M200. Maximum LDH activity was achieved by addition of lysis buffer and normalized by a corresponding volume correction control. Background values (medium only) were subtracted from the sample readings, and the percentage of necrotic cell death was calculated in relation to the maximum LDH release.

TUNEL-based detection of DNA fragmentation. ApoDIRECT In Situ DNA Fragmentation Assay kit was used to detect DNA fragmentation in apoptotic cells though terminal deoxynucleotidyl transferase-mediated dUTP nick end labeling (TUNEL) method. In brief, HT-29 cells were seeded onto a 6 -well plate, at a density of $2 \times 10^{6}$ cells per well and allowed to incubate overnight. Then the cells were treated with various concentrations of imperatorin for $48 \mathrm{~h}$. After a 48-h treatment, the cells were harvested and fixed with $1 \%$ (w/v) paraformaldehyde (PFA) in physiological buffer (PBS). The cells were washed with PBS twice and permeabilized with ice-cold $70 \%$ ethanol in PBS for $\geq 30 \mathrm{~min}$. After fixation, the cells were centrifuged at $500 \mathrm{x} \mathrm{g}$ for $5 \mathrm{~min}$ and the supernatant was decanted. Cell pellets were re-suspended in wash buffer and washed twice prior to a 1-h incubation in staining buffer. After incubation, the stained cells were rinsed twice by adding rinsing buffer, followed by a 30-min incubation in propidium iodide (PI)/RNase A solution in the dark at room temperature. The amount of fluorescein-labeled samples was observed using the FACSVerse system (BD Biosciences, San Jose, CA, USA).
The samples presenting a $\mathrm{FITC}^{+} / \mathrm{PI}^{-}$signal were considered as having fragmented DNA.

Flow cytometric analysis of the cell cycle. HT-29 cells were seeded onto a 6 -well plate, at a density of $1 \times 10^{6}$ cells per well and allowed to incubate overnight. The cells were treated with imperatorin for $48 \mathrm{~h}$. After a 48 -h treatment, the cells were washed with PBS once and harvested with trypsin. The harvested cells were washed with cold PBS twice and fixed with $70 \%$ ethanol for $\geq 1 \mathrm{~h}$. The cells were centrifuged at $500 \mathrm{x}$ g for 5 min and washed twice with cold PBS, followed by addition of PI (final concentration, $40 \mu \mathrm{g} / \mathrm{ml}$ ). The cells were incubated in the dark at $37^{\circ} \mathrm{C}$ for $15 \mathrm{~min}$. Fluorescence-activated cells in each sample were recorded with 10,000 events using the FACSVerse system (BD Biosciences). The cell cycle distribution was analyzed with ModFit (10).

Flow cytometric analysis of apoptosis. HT-29 cells were cultured and treated with imperatorin using the same procedures for the cell cycle analysis. The cells were harvested with trypsin and washed twice with cold PBS. Then the cells were re-suspended in $1 \mathrm{X}$ binding buffer at a density of $1 \times 10^{6}$ cells $/ \mathrm{ml}$. One hundred microliters of cell suspension per sample was transferred to a 5 -ml culture tube and stained with $5 \mu 1$ of FITC Annexin V and $5 \mu \mathrm{l}$ of PI (FITC Annexin V Apoptosis Detection kit I; BD Pharmingen, Franklin Lakes, NJ, USA). The cells were gently vortexed and incubated for $15 \mathrm{~min}$ at room temperature. After incubation, $400 \mu 1$ of $1 \mathrm{X}$ binding buffer was added to each tube. Fluorescence-activated cells in each sample were recorded with 10,000 events by using the FACSVerse System. The cells presenting a $\mathrm{FITC}^{+} / \mathrm{PI}^{\prime}$ signal were considered as early apoptotic cells while the cells presenting with a $\mathrm{FITC}^{+} / \mathrm{PI}^{+}$signal were considered as late apoptotic or dead cells.

Caspase-3/7 assay. The Apo-ONE Homogeneous Caspase-3/7 Assay kit was used to evaluate the activities of apoptosis by assessing the caspase-3/7 enzymatic activities in the imperatorin-treated HT-29 cells. HT-29 cells were seeded onto a 96-well black plate (with clear bottom), at a density of $6 \times 10^{3}$ cells per well and allowed to incubate overnight. Then the cells were treated by either $0.5 \%$ DMSO [as negative control group (Ctrl)] or various concentrations of imperatorin and incubated for $48 \mathrm{~h}$. After incubation, an equal volume of Apo-ONE caspase- $3 / 7$ reagent was added to each well. The plate was incubated for $3 \mathrm{~h}$ at room temperature prior to recording the rate of fluorescence (RFU) of each well at excitation $485 \mathrm{~nm}$ and emission $530 \mathrm{~nm}$ using a TECAN infinite M200.

Western blot analysis. Western blot analysis was carried out following the recommended protocol from Abcam (Cambridge, UK). In brief, HT-29 cells were seeded onto $100-\mathrm{mm}$ culture dishes, at a density of $2 \times 10^{6}$ cells per dish and incubated for $24 \mathrm{~h}$ prior to a 48 -h treatment with various concentrations of imperatorin. After a 48-h treatment, the cells were harvested and lysed using Nonidet-P40 lysis buffer $[150 \mathrm{mM}$ sodium chloride, $1 \%$ Triton X-100, $50 \mathrm{mM}$ Tris, $\mathrm{pH} 8.0$, supplemented with protease inhibitor cocktails (Roche)]. The protein concentration was determined using the DC protein assay (Bio-Rad, Hercules, CA, USA) according to the manufacturer's protocols using bovine serum albumin (BSA) as the 


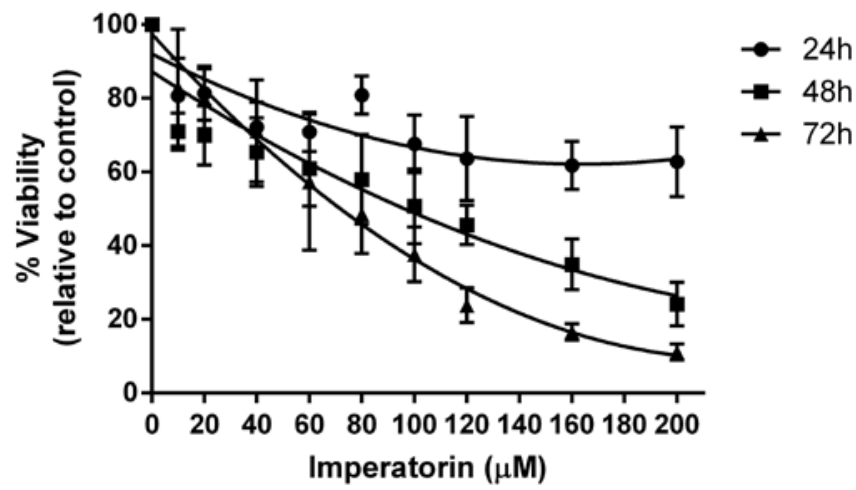

Figure 1. Inhibitory effect of imperatorin on HT-29 cell viability. HT-29 cells were treated with different concentrations of imperatorin for 24, 48 and $72 \mathrm{~h}$, respectively. The cells in the solvent control group were treated with $0.5 \%$ DMSO. Cell viability was determined by MTT assay. Data are presented as mean $\pm \operatorname{SD}(n=3)$.

standard. Normalized protein samples were denatured in a boiling water bath for $10 \mathrm{~min}$ and subjected to sodium dodecyl sulfate polyacrylamide gel electrophoresis (SDS-PAGE) to separate the proteins using $80-120 \mathrm{~V}$ for $2 \mathrm{~h}$. Proteins were transferred from the gel to a $0.25-\mu \mathrm{m}$ PVDF membrane at $12 \mathrm{~V}$ for $30 \mathrm{~min}$ using the Novex Semi-dry blotter (Invitrogen Life Technologies, Carlsbad, CA, USA). Then the membrane was blocked with non-fat dry milk (5\% w/v) in TBST for $1 \mathrm{~h}$ at room temperature before incubation with the specific primary antibody (at appropriate dilution suggested by the product data sheet) in $4 \mathrm{ml}$ of $5 \%$ non-fat milk at $4^{\circ} \mathrm{C}$ overnight. After washing with TBST, the membrane was incubated with the species appropriate HRP-conjugated secondary antibody (at 1:2,000 dilution) in $4 \mathrm{ml}$ of 5\% non-fat milk at room temperature for $1 \mathrm{~h}$. Signals were developed using ECL chemiluminescence detection reagent (GE, Fairfield, CT, USA) and blots were visualized after exposure to Fuji Super RX film. The membrane was re-probed with $\beta$-tubulin as an internal control. The intensity of the blots was quantified by ImageJ image processing program.

ROS assay. Image-iT LIVE Green Reactive Oxygen Species Detection kit is used to detect the production of ROS in live cells. In brief, HT- 29 cells $\left(1 \times 10^{6}\right.$ per well) were seeded on a 6-well plate and allowed to incubate overnight. Then the cells were treated with various concentrations of imperatorin and incubated for $24 \mathrm{~h}$. After a 24-h treatment, the cells were washed with warm Hank's balanced salt solution (HBSS) once before a 25 -min incubation with $25 \mu \mathrm{M}$ carboxy- $\mathrm{H}_{2}$ DCFDA and an additional 5-min incubation with $5 \mu \mathrm{M}$ Hoechst 33342 . The coverslips were gently washed three times with warm HBSS and mounted for fluorescent imaging at excitation $485 \mathrm{~nm}$ and emission $530 \mathrm{~nm}$ by Nikon E80i (Nikon, Tokyo, Japan).

Data analysis. In all viability assays, the viable cells were measured as a percentage relative to the solvent control groups and expressed as means $\pm S D(n \geq 3)$. Nonlinear regression test was applied to the viability assays to obtain a fit curve $\left(\mathrm{R}^{2}>0.98\right)$. Statistical analysis of data was carried out by one-way ANOVA (coupled with post-test, Dunnett's test) to

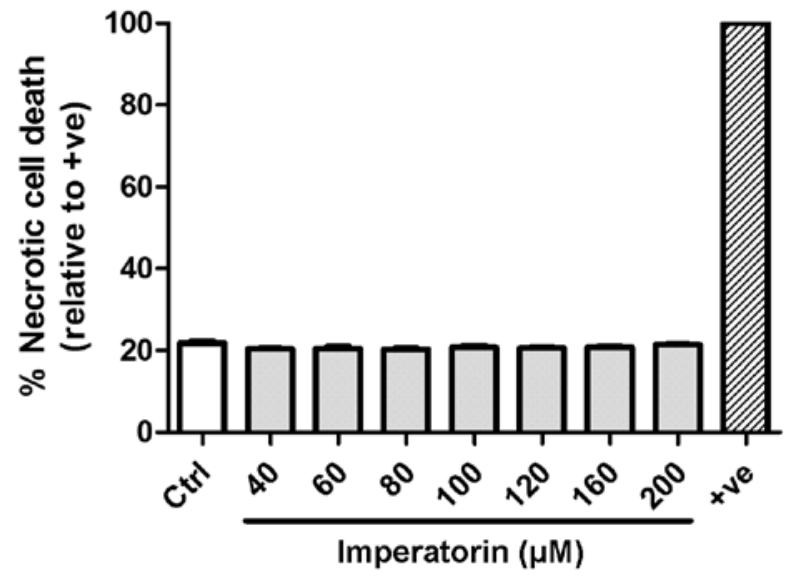

Figure 2. The percentage of necrotic HT-29 cells after treatment of imperatorin. HT-29 cells were treated with different concentrations of imperatorin for $48 \mathrm{~h}$. The cells in the solvent control (Ctrl)/positive control (+ve) groups were treated with $0.5 \% \mathrm{DMSO} / \mathrm{cell}$ lysis buffer. The percentage of necrotic cells was determined by LDH assay. Data are presented as mean \pm SD $(n=3)$.

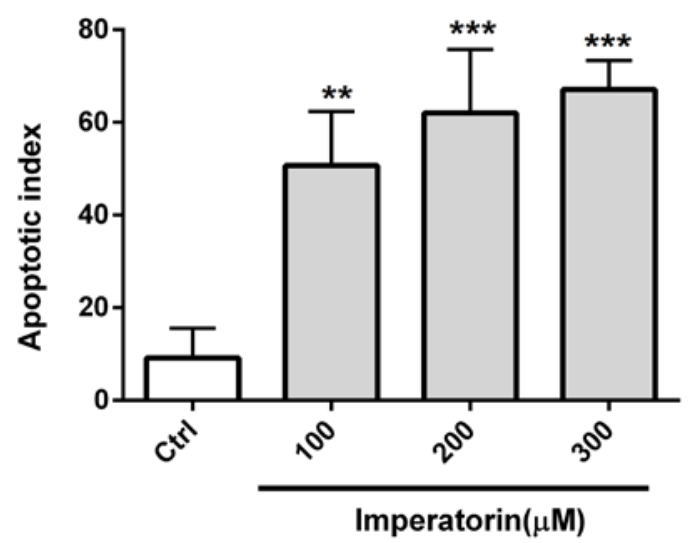

Figure 3. The induction of apoptotic DNA fragmentation in HT-29 cells by imperatorin. HT-29 cells were treated with $0,100,200$ and $300 \mu \mathrm{M}$ imperatorin for $48 \mathrm{~h}$. The cells in the solvent control group were treated with $0.5 \%$ DMSO. The activity of apoptotic DNA fragmentation was evaluated by TUNEL assay. Apoptotic index $=$ (no. of cells with $\left.\mathrm{FITC}^{+} / \mathrm{PI}^{-}\right) /($no. of analyzed cells). Data are presented as mean $\pm \mathrm{SD}(\mathrm{n}=3) ;{ }^{* *} \mathrm{P}<0.01 ;{ }^{* * *} \mathrm{P}<0.005$ vs. the control group

test for differences between the control group and treatment groups. Values of $\mathrm{P}<0.05, \mathrm{P}<0.01, \mathrm{P}<0.005$ and $\mathrm{P}<0.001$ were considered to indicate statistically significant differences and are indicated by asterisks in the figures.

\section{Results and Discussion}

In the present study, different concentrations of imperatorin were used to treat HT-29 cells. An in vitro study on HT-29 colon cancer cells revealed that imperatorin inhibited cell viability with an $\mathrm{IC}_{50}$ value of $239 \mu \mathrm{M}$ after $24 \mathrm{~h}, 101 \mu \mathrm{M}$ after $48 \mathrm{~h}$ and $78 \mu \mathrm{M}$ after $72 \mathrm{~h}$ of incubation (Fig. 1). The percentage of necrotic cell death remained unaffected with an increase in imperatorin concentration (Fig. 2). The results suggest that imperatorin did not cause necrosis of HT-29 cells. In contrast, the apoptotic index which is used to determine cancer cell growth and is inversely related to necrosis showed 

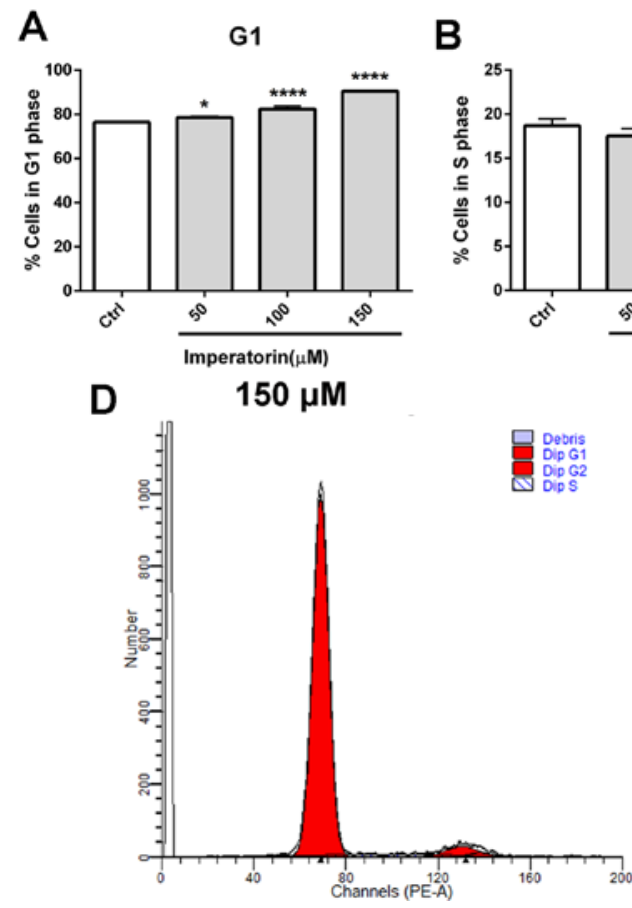

s

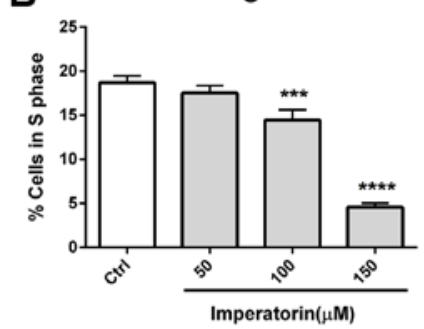

E
C

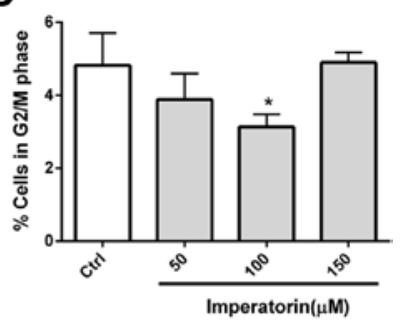

Ctrl

Figure 4. The induction of cell cycle arrest in HT-29 cells by imperatorin. HT-29 cells were treated with different concentrations of imperatorin for $48 \mathrm{~h}$. The cells in the solvent control group were treated with $0.5 \%$ DMSO. The percentage of cells in the G1, S and G2/M phases of the cell cycle was determined by propidium iodide (PI) staining and observed by flow cytometry (A-C). Typical distribution of the cell populations in cells treated with $150 \mu \mathrm{M}$ imperatorin and control cells is shown (D and $\mathrm{E}$, respectively). Data are presented as mean $\pm \mathrm{SD}(\mathrm{n}=3) ;{ }^{*} \mathrm{P}<0.05 ;{ }^{* * *} \mathrm{P}<0.005 ;{ }^{* * * *} \mathrm{P}<0.001$ vs. the control group.
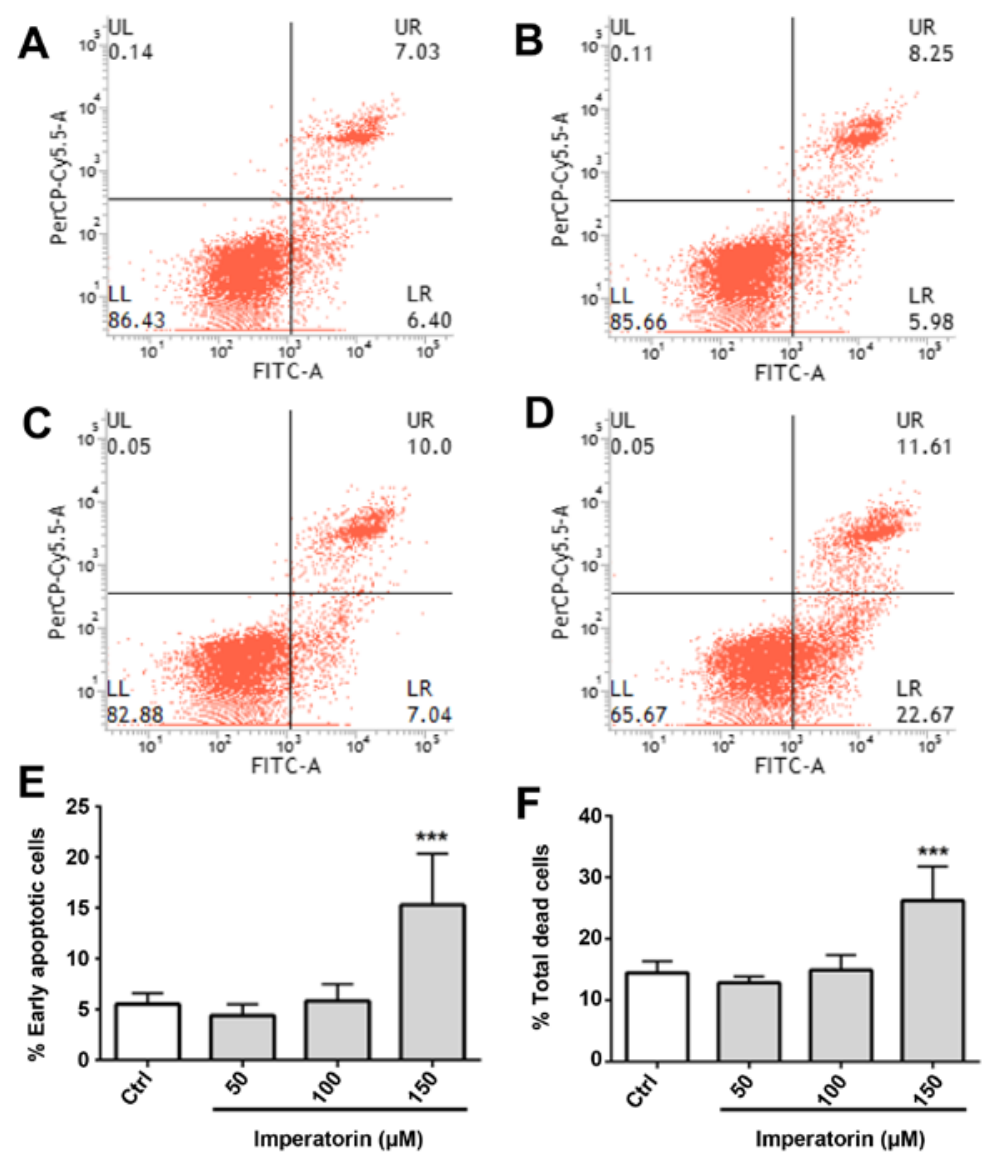

Figure 5. The induction of apoptosis in the HT-29 cells by imperatorin. HT-29 cells were treated with different concentrations of imperatorin for $48 \mathrm{~h}$. The cells in the solvent control group were treated with 0.5\% DMSO. The percentage of apoptotic cells was determined by Annexin V-FITC/PI staining and observed by flow cytometry. The cells generating an $\mathrm{FITC}^{+} / \mathrm{PI}(\mathrm{LR})$ signal were in the early apoptotic stage while the cells generating an $\mathrm{FITC}^{+} / \mathrm{PI}^{+}(\mathrm{UR})$ signal were dead cells. Typical distribution of cell population following the treatment of $0,50,100$ and $150 \mu \mathrm{M}$ imperatorin is shown (A-D, respectively). The percentage of early apoptotic cells and total dead cells $(\mathrm{LR}+\mathrm{UR})$ is shown $\left(\mathrm{E}\right.$ and $\mathrm{F}$, respectively). Data are presented as mean $\pm \mathrm{SD}(\mathrm{n}=3) ;{ }^{* * * *} \mathrm{P}<0.005 \mathrm{vs}$. control group. 


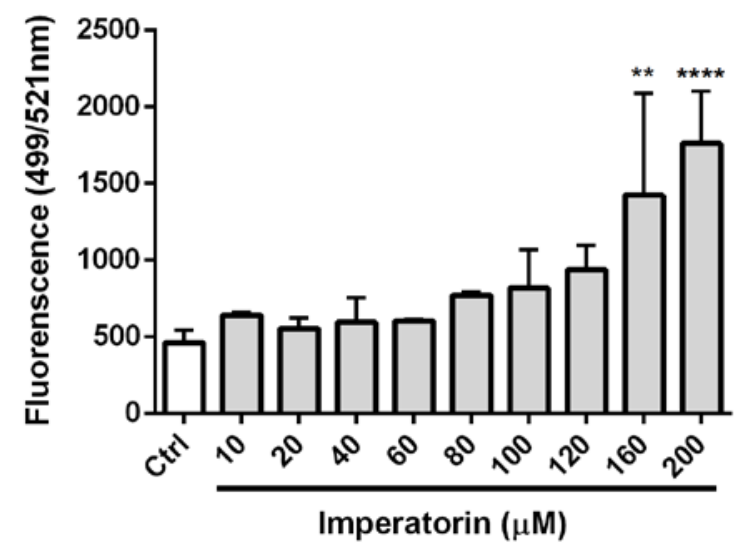

Figure 6. Caspase-3/7 activities in the HT-29 cells after treatment with imperatorin. HT-29 cells were treated with different concentrations of imperatorin for $48 \mathrm{~h}$. The cells in the solvent control group were treated with $0.5 \%$ DMSO. The caspase-3/7 activities in HT-29 cells were evaluated by the caspase-3/7 assay. Data are presented as mean $\pm \mathrm{SD}(\mathrm{n}=3) ;{ }^{* *} \mathrm{P}<0.01$; ${ }^{* * * * *} \mathrm{P}<0.001$ vs. control group.

a concentration-dependent increase in the HT-29 cells (Fig. 3). The results indicate that imperatorin induced programmed cell death and provide supportive evidence for an imperatorin-induced apoptotic mechanism that could be mediated through different apoptotic activities (11). The duration of the apoptotic activity was largely dependent on the concentration of the anti-proliferative agent. The common technique that is adopted for measuring the apoptotic index is flow cytometry. Cell cycle analysis showed that imperatorin induced cell cycle arrest at the G1 phase (Figs. 4 and 5). Apoptotic cell death can result from activation of interrelated molecular cascades. The extrinsic pathway transduces extracellular stimulus through the plasmatic membrane. In contrast, the intrinsic pathway modulates the intracellular environment that controls the survival or death of cells. Mitochondria play an important role in controlling the signaling pathways leading to death or survival of cells; thus, anti-proliferative agents can induce oxidative stress and alterations in the mitochondrial membrane that leads to apoptosis activation. These two pathways translate into a convergent point where caspases downregulate the apoptotic activities. The intrinsic apoptotic pathway involves the mitochondria and activation of the caspase cascade which was triggered by imperatorin (Fig. 6). The caspase-3/7 activities in the HT-29 cells after treatment with imperatorin showed a considerable increase. Stimulation of the intrinsic apoptotic pathway by imperatorin triggered a signaling cascade that resulted in the increase of the apoptotic process with caspase-3/7, which were activated with the increasing concentration of imperatorin. These data demonstrate the anti-proliferative activity of imperatorin in HT-29 cells. The fluorescence values for caspase- $3 / 7$ provided a sensitive approach to analyze the apoptotic and anti-proliferative effect of imperatorin treatment. The results showed that imperatorin activated caspases resulting in enhancing caspase activity (Fig. 6). The activation of this intrinsic pathway was accompanied by Cyt-C and the caspase cascade (Fig. 8).

The Bcl-2 family members exhibit a regulatory role in determining cell viability. These members exert checkpoints to modulate apoptotic activities. The ratio between

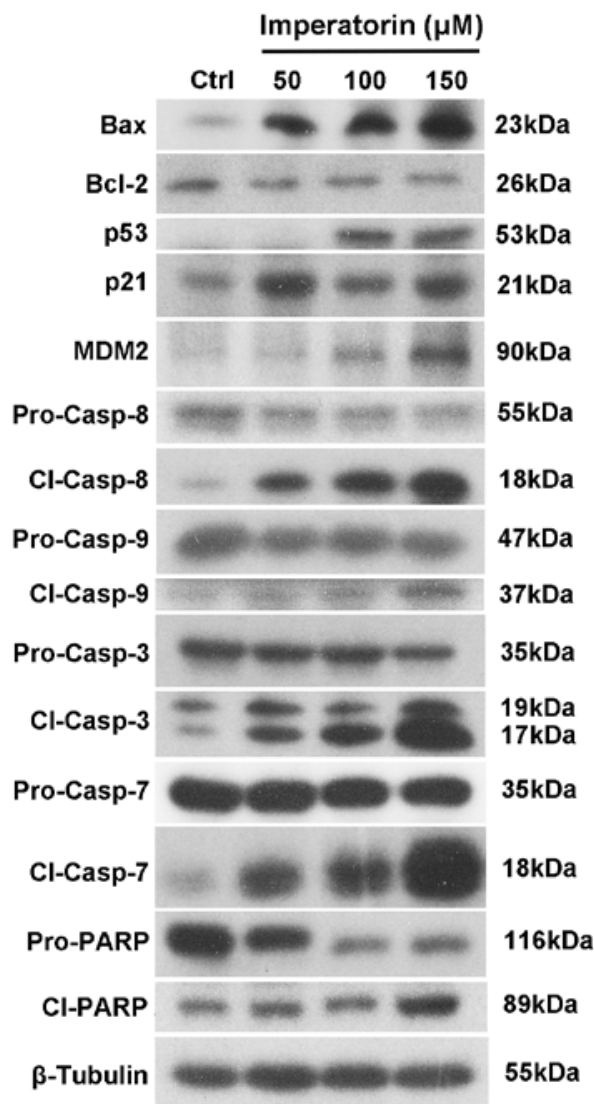

Figure 7. Expression of apoptosis-related proteins in HT-29 cells after treatment of imperatorin. HT-29 cells were treated with different concentrations of imperatorin for $48 \mathrm{~h}$. The cells in the solvent control group were treated with $0.5 \%$ DMSO. Western blot analysis was conducted with antibodies against mitochondrial-mediated apoptotic proteins: Bax and Bcl-2; death-receptor-mediated apoptotic proteins: p53, p21 and MDM2; proteins in caspase-dependent apoptotic signaling pathways: pro-caspase-8, cleaved caspase- 8 , pro-caspase- 9 , cleaved caspase- 9 , pro-caspase- 3 , cleaved caspase-3, pro-caspase-7, cleaved caspase-7, PARP and cleaved PARP.

pro-apoptotic factors including Bax, Bad, Bak, Bid, MDM2 and anti-apoptotic members is a useful marker for measuring apoptosis. Changes in expression of Bcl-2 is indicative of the alteration of mitochondrial membrane potential. Fig. 7 shows that imperatorin modulated the alteration of the expression of these key apoptotic members in the HT-29 cells. Other key molecules involved in apoptosis regulation is alteration of the transcription factor p53 (Fig. 8). The main role of p53 is to mediate tumorigenesis (12). Cancer involves complex cellular changes which are associated with uncontrollable replication and resistance to growth-controlled mechanisms.

Malignant tumors may metastasize through the circulatory system. Consequently, metastasis causes significant tumor burden. At present, strategies of cancer treatment using phytochemicals in combination with targeted therapies are considered promising, and may lead to greater efficacy and enhanced patient survival rates (13). Malignant cells may find alternative survival mechanisms. Indeed, imperatorin is capable of modulating cell conditions that may cause alteration in expression profile of oncogenes and cell cycle mechanisms of cancer cells. Anti-proliferative agents can consequently be used to suppress cancer cell survival or death based on the modulation of the intrinsic pathway. Imperatorin was shown to 
A

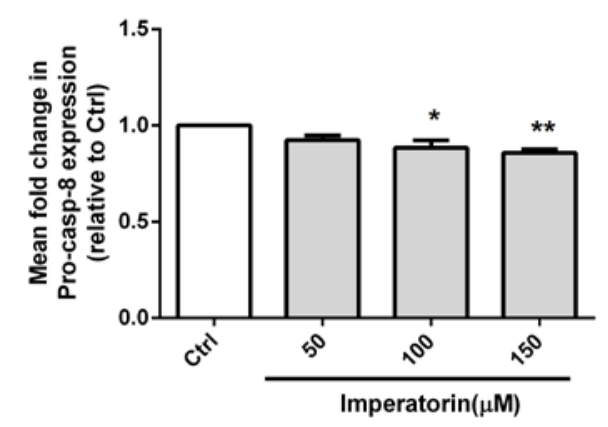

C

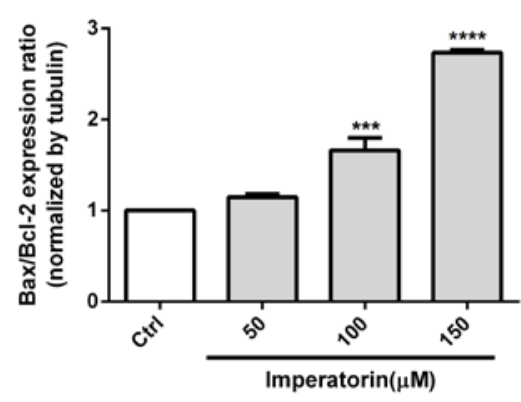

$\mathbf{E}$

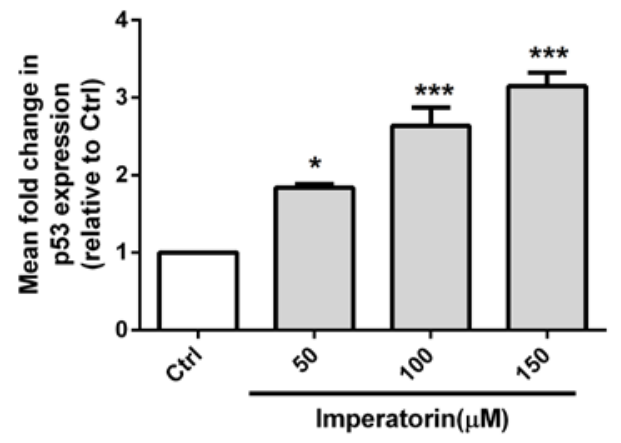

G

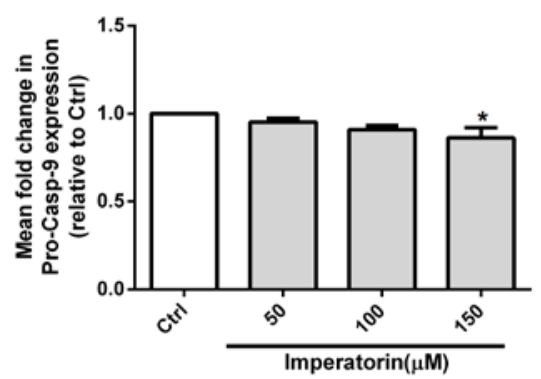

I

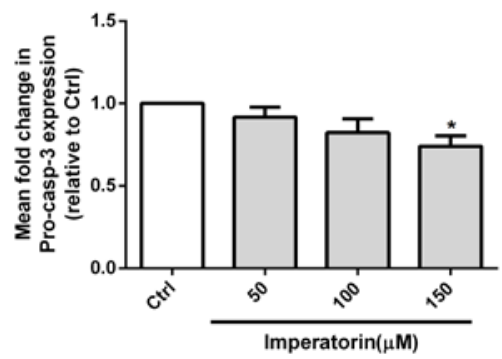

B

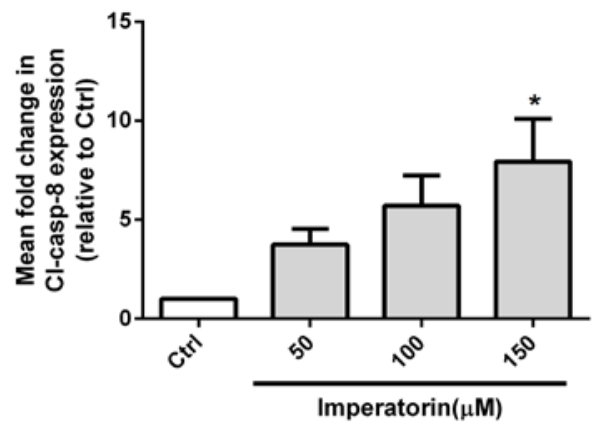

D

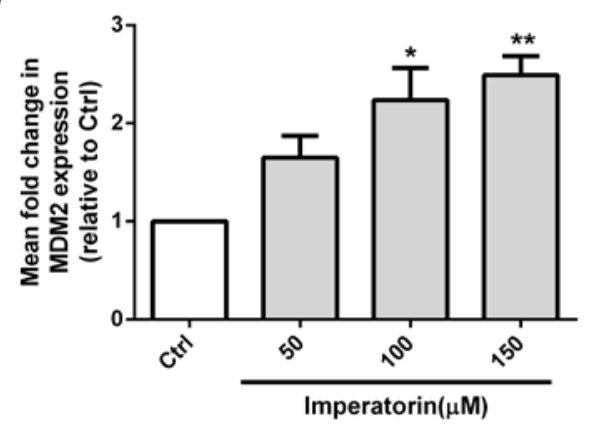

$\mathbf{F}$

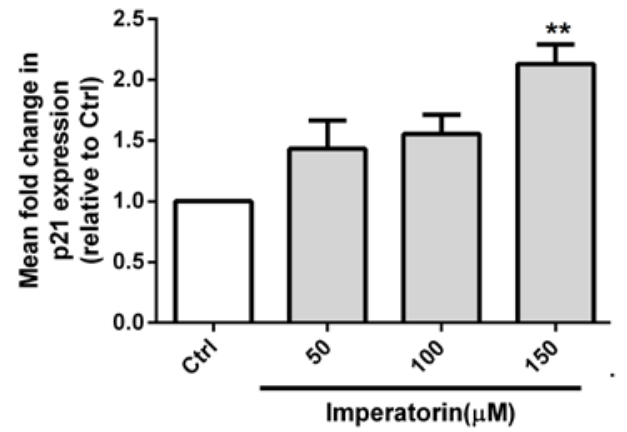

H

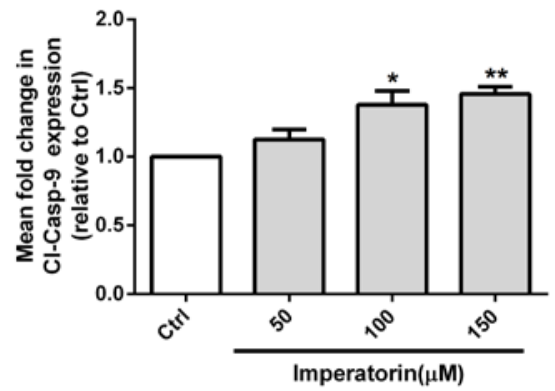

$\mathbf{J}$

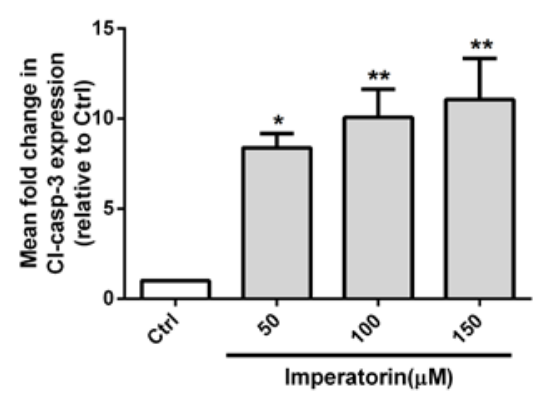

Figure 8. (A-J) Expression levels of proteins were quantified by ImageJ image processing program. Particularly, the Bax/Bcl-2 expression ratio is shown in C. $\beta$-tubulin was used as an internal control to normalize data. Data are presented as mean $\pm \mathrm{SD}(\mathrm{n}=3) ;{ }^{*} \mathrm{P}<0.05 ;{ }^{* *} \mathrm{P}<0.01 ;{ }^{* * * *} \mathrm{P}<0.005 ;{ }^{* * * *} \mathrm{P}<0.001$ vs. the control group. 

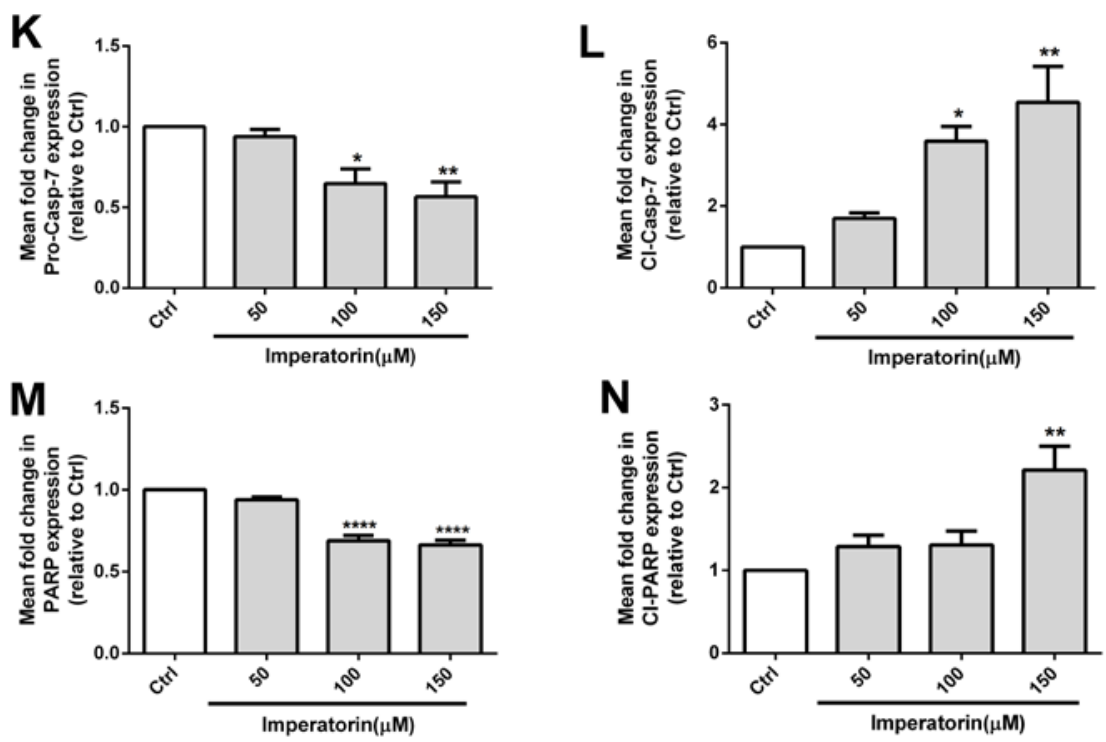

Figure 8. (Continued). (K-N) The expression levels of the proteins were quantified by ImageJ image processing program. $\beta$-tubulin was used as an internal control to normalize data. Data are presented as mean $\pm \mathrm{SD}(\mathrm{n}=3) ;{ }^{*} \mathrm{P}<0.05 ;{ }^{* * *} \mathrm{P}<0.01 ;{ }^{* * * *} \mathrm{P}<0.005 ;{ }^{* * * * *} \mathrm{P}<0.001$ vs. control group.

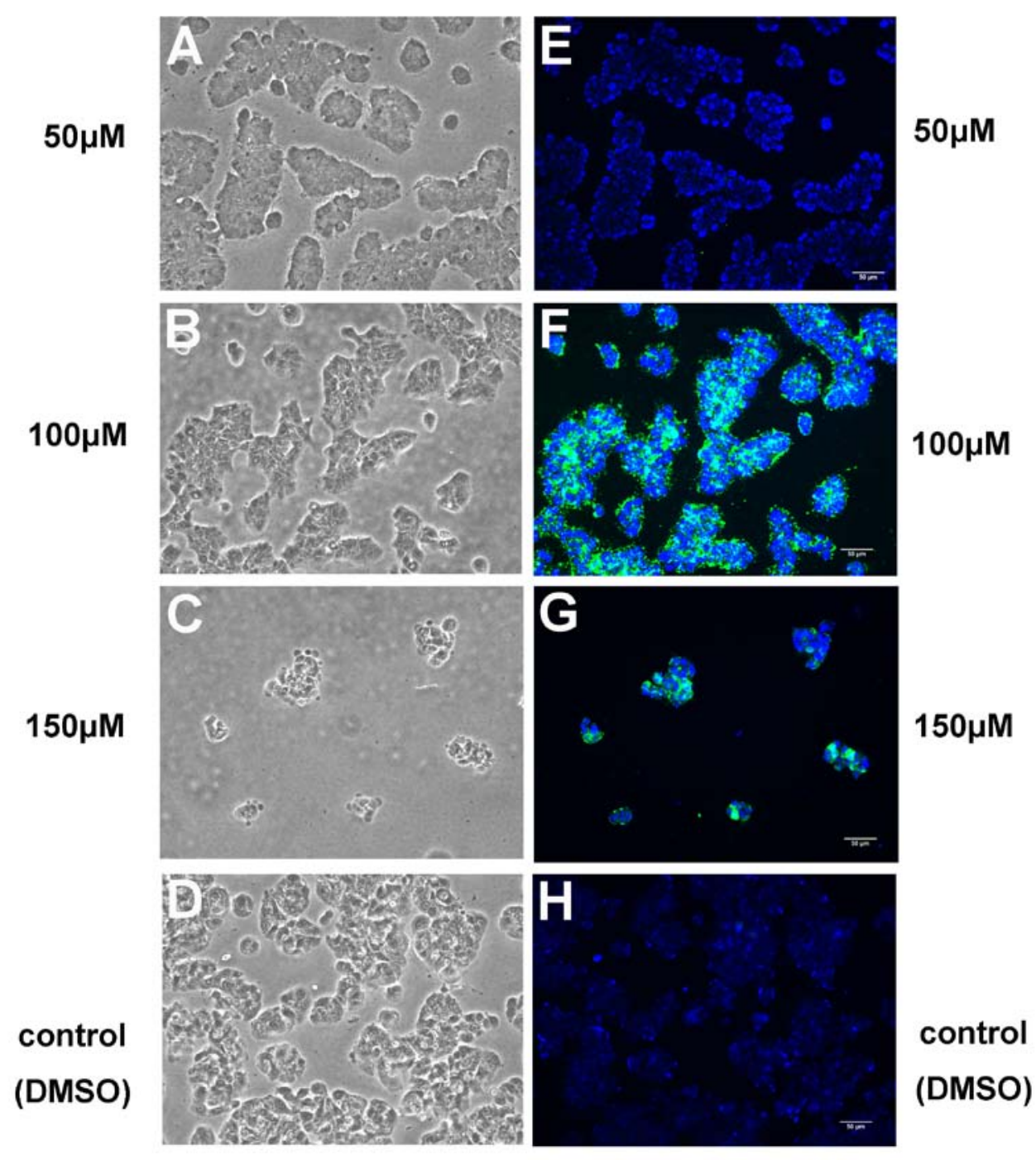

Figure 9. The production of reactive oxygen species (ROS) in HT-29 cells after treatment with imperatorin. HT-29 cells were treated with different concentrations of imperatorin for $48 \mathrm{~h}$. The cells in the solvent control group were treated with $0.5 \%$ DMSO. The generation of ROS in imperatorin-treated cells was detected by an ROS detection kit. Imperatorin-treated cells were imaged under a bright-field microscope (A-D) as well as a fluorescence microscope (E-H). Intracellular ROS are indicated in green. 
modulate the intrinsic pathway involving Bcl-2 members, $\mathrm{p} 53$, p21, MDM2 and the caspase cascade (Fig. 8), and triggered considerable changes in Bax, caspase-8, MDM2, p53 and p21 expression (Fig. 8).

The maintenance of homeostasis is known to be important in normal tissues that require a balance between cell proliferation and cell death. Bcl-2 family members play a significant role in the regulation of cell death. Bcl-2 is involved in programmed cell death. Overexpression of Bcl-2 in cells results in extended cell survival. Bcl-2 does not appear to affect the generation of reactive oxygen radicals but may prevent oxidative damage to cellular constituents. A pre-set ratio of Bcl-2/Bax appears to determine the cell survival or death of cells after an apoptotic stimulus (14). The increase in $\mathrm{Bax} / \mathrm{Bcl}-2$ expression following incubation of the HT-29 cells with imperatorin suggests induction of apoptosis of HT-29 cancer cells. The overexpression of p53 and p21 provided supportive evidence of apoptosis (Fig. 8).

The anti-proliferative activity of imperatorin was demonstrated in the present study. The alteration of gene expression in imperatorin-treated HT-29 cells revealed that imperatorin induced apoptosis via a p53-dependent pathway. The results are analogous with in vitro studies in HepG2 cells (1). The production of reactive oxygen species (ROS) in HT-29 after treatment with imperatorin was examined using an ROS detection kit. Imperatorin increased the ROS levels in the HT-29 cells but not in normal cells (3). However, there is little information concerning the selectivity of imperatorin. The cellular changes were examined under bright-field microscope and fluorescence microscope (Fig. 9). The results showed that the level of ROS was increased with increasing concentrations of imperatorin. One of the advantages of staining methodology is the ability to show the qualitative analysis with both phase contrast and fluorescent images. The classical morphological changes associated with apoptosis include cell shrinkage, membrane blebbing, nuclear condensation, and DNA fragmentation. The microscopic examination of the cellular changes presented in Fig. 8 highlights this advantage, illustrating the ability to use phase contrast and fluorescent blended images to correlate the activation of caspase- $3 / 7$ and the disintegration of nuclei due to cell death that showed morphological changes in response to treatment with imperatorin. The results significantly enhance the confidence in the measured apoptotic activities and conclusions drawn from the image analysis. The resulting data revealed the effectiveness of imperatorin as an anti-proliferative agent.

In conclusion, the results demonstrated the anti-proliferative activities of imperatorin in the HT-29 cell line. Imperatorin induced apoptosis via the activation of caspase-3/7, a primary and irreversible executioner pathway in HT-29 cancer cells. The fluorescent labeling assay demonstrated the inhibitory effects of imperatorin on HT-29 cell proliferation. The results provide confirmative evidence of apoptotic signaling in HT-29 cells.
The results were validated by individual images. Together, these results provide experimental evidence for apoptotic signaling after treatment of HT-29 cells with imperatorin. The anti-proliferative activities of imperatorin in HT-29 cells were demonstrated in the present study.

\section{Acknowledgements}

Professor Yi Mei Zheng gratefully acknowledged the support by the Training Program of FuJian Excellent Talents in University in China. The present study was supported in part by a grant no. 6903088 from CUHK.

\section{References}

1. Rahman A, Siddiqui SA, Jakhar R and Kang SC: Growth inhibition of various human cancer cell lines by imperatorin and limonin from Poncirus trifoliata Rafin. seeds. Anticancer Agents Med Chem 15: 236-241, 2015.

2. Kumar D, Bhat ZA, Kumar V and Shah MY: Coumarins from Angelica archangelica Linn. and their effects on anxiety-like behavior. Prog Neuropsychopharmacol Biol Psychiatry 40: 180-186, 2013

3. Sigurdsson S and Gudbjarnason S: Effect of oral imperatorin on memory in mice. Biochem Biophys Res Commun 441: 318-320, 2013.

4. Sigurdsson S, Ogmundsdottir HM and Gudbjarnason S: Antiproliferative effect of Angelica archangelica fruits. Z Naturforsch C 59: 523-527, 2004.

5. Luszczki JJ, Glowniak K and Czuczwar SJ: Imperatorin enhances the protective activity of conventional antiepileptic drugs against maximal electroshock-induced seizures in mice. Eur J Pharmacol 574: 133-139, 2007.

6. Sancho R, Márquez N, Gómez-Gonzalo M, Calzado MA, Bettoni G, Coiras MT, Alcamí J,López-Cabrera M, Appendino G and Muñoz E: Imperatorin inhibits HIV-1 replication through an Sp1-dependent pathway. J Biol Chem 279: 37349-37359, 2004.

7. Appendino G, Bianchi F, Bader A, Campagnuolo C, Fattorusso E, Taglialatela-Scafati O, Blanco-Molina M, Macho A, Fiebich BL, Bremner P, et al: Coumarins from Opopanax chironium. New dihydrofuranocoumarins and differential induction of apoptosis by imperatorin and heraclenin. J Nat Prod 67: 532-536, 2004.

8. Kim YK, Kim YS and Ryu SY: Antiproliferative effect of furanocoumarins from the root of Angelica dahurica on cultured human tumor cell lines. Phytother Res 21: 288-290, 2007.

9. Wu KC, Chen YH, Cheng KS, Kuo YH, Yang CT, Wong KL, Tu YK, Chan P and Leung YM: Suppression of voltage-gated $\mathrm{Na}(+)$ channels and neuronal excitability by imperatorin. Eur $\mathrm{J}$ Pharmacol 721: 49-55, 2013.

10. Allen GD: MODFIT: a pharmacokinetics computer program. Biopharm Drug Dispos 11: 477-498, 1990.

11. Potten CS: What is an apoptotic index measuring? A commentary. Br J Cancer 74: 1743-1748, 1996.

12. Lavin MF and Gueven N: The complexity of p53 stabilization and activation. Cell Death Differ 13: 941-950, 2006.

13. Mitsiades CS, Davies FE, Laubach JP, Joshua D, San Miguel J, Anderson KC and Richardson PG: Future directions of next-generation novel therapies, combination approaches, and the development of personalized medicine in myeloma. J Clin Oncol 29: 1916-1923, 2011.

14. Korsmeyer SJ, Shutter JR, Veis DJ, Merry DE and Oltvai ZN: Bcl-2/Bax: A rheostat that regulates an anti-oxidant pathway and cell death. Semin Cancer Biol 4: 327-332, 1993. 